\title{
Factors affecting the outcomes in patients with acute respiratory distress syndrome in a tertiary care setting
}

\author{
Muhammed Rashid ${ }^{\mathrm{a}}$, Manasvini Ramakrishnan ${ }^{\mathrm{a}}$, Deepa Sudalai Muthu ${ }^{\mathrm{a}}$, \\ Viji Pulikkel Chandran ${ }^{a}$, Girish Thunga ${ }^{a}$, Vijayanarayana Kunhikatta ${ }^{\mathrm{a}}$, Vishal Shanbhag ${ }^{\mathrm{b}}$, \\ Raviraja V. Acharya ${ }^{c}$, Sreedharan Nair ${ }^{\text {a, }}$, \\ ${ }^{a}$ Department of Pharmacy Practice, Manipal College of Pharmaceutical Sciences, Manipal Academy of Higher Education, Manipal, 576104, India \\ ${ }^{\mathrm{b}}$ Department of Critical Care Medicine, Kasturba Medical College, Manipal Academy of Higher Education, Manipal, 576104, India \\ ${ }^{\mathrm{c}}$ Department of Medicine, Kasturba Medical College, Manipal Academy of Higher Education, Manipal, Karnataka, 576104, India
}

\section{A R T I C L E I N F O}

\section{Keywords:}

ARDS

Predictors

Recovery

Sepsis

India

\begin{abstract}
A B S T R A C T
Purpose: The clinical profile and factors affecting outcomes in acute respiratory distress syndrome (ARDS) from adequately sample-sized Indian studies are still lacking. We aimed to investigate the clinical profile, treatment pattern, outcomes; and to assess factors affecting non-recovery in ARDS patients.

Patients and methods: A retrospective observational study was conducted among adult ARDS patients admitted during five year period (January 2014-December 2018) in a South Indian tertiary care setting. The relevant data were collected from the medical records to the data collection form. The univariate and multivariate logistic regression analyses were conducted to identify the predictors of outcomes using SPSS v20.

Results: A total of 857 participants including 496 males and 361 females with a mean age of $46.86 \pm 15.81$ years were included in this study. Fever (70.9\%), crepitation (58.3\%), breathlessness $(56.9 \%)$, and cough (45\%) were the major clinical presentation. Hypertension (25.2\%), kidney disease (23.8\%), and diabetes (22.3\%) were the major comorbidities; and sepsis (37.6\%), pneumonia (33.3\%), and septic shock (27.5\%) were the major etiological factors observed. Antibiotics and steroids were administered to $97.9 \%$ and $52.3 \%$ of the population, respectively. The recovery rate was $47.49 \%$. The patients with scrub typhus, dengue, pancreatitis, and oxygen supplementation had significantly lower mortality. The factors such as advanced age, sepsis, septic shock, liver diseases, and ventilation requirements were observed to be the independent predictors of non-recovery in ARDS patients.

Conclusion: A comparable recovery rate was observed in our population. Advanced age, sepsis, septic shock, liver diseases, and ventilation requirements were the independent predictors of non-recovery.
\end{abstract}

\section{Introduction}

American-European Consensus Conference (AECC) criteria 1994 says that acute respiratory distress syndrome (ARDS) is acute damage of lungs with an arterial oxygen partial pressure to fractional inspired oxygen ( $\mathrm{PiO} 2 / \mathrm{FiO} 2)$ ratio of less than $200 \mathrm{mmHg}$ regardless of positive end-expiratory pressure (PEEP) level, which can be observed by a bilateral infiltrate on the frontal chest radiograph with a pulmonary artery wedge pressure of $<18 \mathrm{mmHg}$ without evidence of left-sided hypertension or heart failure. ${ }^{1}$ Later Berlin definition in 2012 recommends the following, i) the onset should be within a week time; ii) presence of bilateral opacities in the chest imaging which is not explained by effusions, collapse, or the nodules; iii) respiratory edema which is not explained by cardiac failure; iv) with a $\mathrm{PaO} 2 / \mathrm{FiO} 2$ ratio of less than $200 \mathrm{mmHg}^{2}$

ARDS is a global threat with significant health and economic burden

; ARDS, Acute Respiratory Distress Syndrome; AECC, American-European Consensus Conference; CI, Confidence Interval; COVID-19, Coronavirus Disease; ICU, Intensive Care Unit; IEC, Institutional Ethics Committee; MODS, Multiple Organ Dysfunction Syndrome; OR, Odds Ratio; PEEP, Positive end expiratory pressure; PiO2/FiO2, Arterial oxygen partial pressure tofractional inspired oxygen; RCT, Randomized Controlled Trial; SARS-CoV 2, Severe Acute Respiratory Syndrome Coronavirus 2.

* Corresponding author.

E-mail address: nair.sreedhar@manipal.edu (S. Nair). 
as it needs intensive medical and pharmaceutical care. Incidence differs from location to location, which ranged from 1.5 cases to 79 cases per 100000 inhabitants. ${ }^{3}$ Indian studies reported an $11.4 \%$ incidence rate of ARDS among the ventilated patients and $41.8 \%$ of mortality among the ARDS patients ${ }^{4}$ whereas, Sharma et al., reported $56.2 \%$ of mortality rate in 28-days. ${ }^{5}$

The major risk factors for the development of ARDS include pneumonia, sepsis, gastric and/or oral and oesophageal contents aspiration, trauma, poison, acute pancreatitis, transfusion of blood and blood products, medication overdose, near drowning, and smoke inhalation., Whereas the risk factors such as age, trauma, oxygenation index, $\mathrm{PaO} 2 / \mathrm{FiO} 2$ ratio, $\mathrm{FiO} 2$, and $\mathrm{PaO} 2$ level, blood $\mathrm{pH}$, liver cirrhosis, non-pulmonary organ failure, Acute Physiology Score, peak pressure were the significant predictors for mortality in ARDS patients. ${ }^{5,7,8}$

The management strategies for ARDS include respiratory supports, prone positioning, administration of neuromuscular blockade, noninvasive oxygenation, fluid management, rescue therapies, and medical management depending on the co-morbidities and other complications in the patients. ${ }^{6}$

All this evidence indicates the epidemiological burden of ARDS is still high across the globe even in this era of modern medicine. Moreover, clinical data on the epidemiological pattern and factors affecting the outcomes in ARDS patients from developing countries like India still lacking. Moreover, the inadequate sample size in all of the published research, which ranged from the fifties to the hundreds, is a major issue .4 This study aimed to investigate the clinical profile, treatment pattern, and outcomes in adult patients with ARDS through a retrospective analysis of five-year data and also to assess the factors affecting non-recovery among this cohort of patients.

\section{Methodology}

\subsection{Ethical consideration}

This retrospective study was carried out after obtaining ethical approval from the relevant institutional ethics committee (IEC 549/ 2019).

\subsection{Study setting and participants}

A retrospective observational study was conducted in a tertiary care hospital located in South India, Karnataka. The data were collected from the medical records of the patients who are diagnosed with ARDS (ICD.10 code: J-80) during January 2014 to December 2018. The adult patients aged 18 years or above and diagnosed with ARDS according to the clinician's judgment were included without any gender restrictions.

\subsection{Data sample}

A total of 921 participants were identified, out of which 64 were excluded as they were aged less than 18 years, and the remaining 857 ARDS patients were included in this study.

\subsection{Data collection and tools}

The parameters such as patient demographics, admission details, signs and symptoms, co-morbidities, laboratory investigations, treatment characteristics, treatment outcome, and duration of hospitalization, ICU, and ventilation were collected to a case record form and it was transcribed to a password-protected laptop. The normal values of the laboratory parameters were considered as per the hospital in which the study was conducted to avoid mismatches.

\subsection{Outcomes considered}

The primary endpoint was recovery. The patients were categorized into two groups, in which those patients who are completely recovered and discharged were considered as recovery group and those who were dead or discharged against the medical advice were considered as the non-recovery group. The secondary endpoints include duration of hospitalization, ICU stay, ventilation days, oxygenation days, and antibiotics days.

\subsection{Statistical analysis}

The statistical analysis was performed using Statistical Package for the Social Sciences version 20.0 developed by IBM. ${ }^{9}$ Descriptive statistics were performed and the results were presented as the frequency with percentages in case of categorical variables and mean (standard deviation) or median (interquartile range) in case of continuous variables, respectively. Following the exploratory analysis, a univariate logistic regression was used for preliminary identification of factors affecting non-recovery in ARDS patients. Factors showing significant association with non-recovery were used in multiple logistic regression for the identification of independent risk factors associated with non-recovery. The results of univariate and multivariate logistic regression were presented in terms of odds ratio (OR) along with its $95 \%$ confidence interval (CI). A probability of less than 0.05 was considered to be statistically significant.

\section{Results}

\subsection{Demographic characteristics of included participants}

A total of 857 participants including 496 (57.9\%) male and 361 (42.1\%) females with a mean age of $46.86 \pm 15.81$ years were included. Out of which, 407 were recovered which indicates $47.49 \%$ of recovery rate with ARDS. The mean age for non-recovered patients was higher $(49.08 \pm 16.57$ years) than the recovered patients $(44.41 \pm 14.53$ years). Among the included participants, 33 (3.9\%) were smokers and 393 (45.9\%) were non-smokers, however, the remaining 431 (50.3\%) patients' smoking status was not available from the data. Similarly, 83 (9.7\%) patients were alcoholics, $352(41.1 \%)$ were non-alcoholics, and the remaining $422(49.2 \%)$ couldn't be identified (Table 1 ).

\subsection{Clinical manifestations, comorbidities, and risk factors}

Majority of the participants observed to have fever $(n=608 ; 70.9 \%)$, followed by crepitation ( $\mathrm{n}=500 ; 58.3 \%$ ), breathlessness $(\mathrm{n}=488$; $56.9 \%)$ and cough $(\mathrm{n}=386 ; 45 \%)$ as a clinical presentation. $25.2 \%$ $(\mathrm{n}=216)$ had hypertension, $23.8 \%(\mathrm{n}=204)$ had kidney disease, and $22.3 \%(n=191)$ had diabetes, respectively. A majority $(n=322 ; 37.6 \%)$ of the included patients had sepsis as a risk factor for ARDS, followed by, pneumonia $(n=285 ; 33.3 \%)$, septic Shock $(n=236 ; 27.5 \%)$, and multiorgan dysfunction syndrome (MODS) $(n=126 ; 14.7 \%)$ were the other major risk factors. A detailed demographic characters of the included participants, recovered and non-recovered group is presented in Table 1.

\subsection{Laboratory parameters among the included participants}

\subsubsection{Vitals}

The average vitals were observed to be near normal, which includes a temperature of $98.82 \pm 0.80 \mathrm{~F}$, heart rate of $98 \pm 22$ beats per minute, systolic blood pressure of $121.49 \pm 25.36 \mathrm{mmHg}$, and diastolic blood pressure of $76.04 \pm 12.56 \mathrm{mmHg}$. Whereas, respiratory rate $(26.10 \pm$ 8.94 breaths per minute) was observed to be higher than normal (Table 2).

\subsubsection{Blood indices}

The average levels of blood indices such as eosinophils count (1.04 \pm 2.43), basophils $(0.57 \pm 1.68)$, and monocytes $(5.88 \pm 4.42)$ were within the normal. Whereas, the total leukocyte count (12408.81 \pm 
Table 1

Demographic characteristics of included participants.

\begin{tabular}{|c|c|c|c|}
\hline Parameter & $\begin{array}{l}\text { Total participants } \\
(\mathrm{N}=857)\end{array}$ & $\begin{array}{l}\text { Recovery group } \\
(\mathrm{n}=407)\end{array}$ & $\begin{array}{l}\text { Non-recovery } \\
\text { group }(n=450)\end{array}$ \\
\hline Age (Mean, SD) & $46.86(15.81)$ & $44.41(14.53)$ & $49.08(16.57)$ \\
\hline Age (Median, IQR) & $47(35,59)$ & $45(32,55)$ & $50(36,62)$ \\
\hline \multicolumn{4}{|l|}{ Gender (n, \%) } \\
\hline Male & $496(57.9)$ & $213(52.34)$ & $283(62.89)$ \\
\hline Female & $361(42.1)$ & $194(47.66)$ & $167(37.11)$ \\
\hline \multicolumn{4}{|l|}{ Smoking status (n, \%) } \\
\hline Yes & $33(3.9)$ & $14(3.45)$ & $19(4.22)$ \\
\hline No & $393(45.9)$ & $194(47.66)$ & $199(44.22)$ \\
\hline Not Reported & $431(50.3)$ & 199 (48.89) & $232(51.55)$ \\
\hline \multicolumn{4}{|l|}{ Alcoholic status (n, \%) } \\
\hline Yes & $83(9.7)$ & $39(9.58)$ & $44(9.78)$ \\
\hline No & $352(41.1)$ & $175(43.00)$ & $177(39.33)$ \\
\hline Not Reported & $422(49.2)$ & $193(47.42)$ & $229(50.89)$ \\
\hline \multicolumn{4}{|l|}{ Comorbidity (n, \%) } \\
\hline Yes & $535(62.4)$ & $231(56.76)$ & $304(67.55)$ \\
\hline No & $322(37.6)$ & $176(43.24)$ & $146(32.45)$ \\
\hline \multicolumn{4}{|l|}{ Risk factor (n, \%) } \\
\hline Yes & $812(94.7)$ & 389 (95.58) & $423(94)$ \\
\hline No & $45(5.3)$ & $18(4.42)$ & $27(6)$ \\
\hline \multicolumn{4}{|c|}{ Signs and Symptoms (n, \%) } \\
\hline Fever & 608 (70.9) & 334 (82.06) & 274 (60.89) \\
\hline Cough & $386(45.0)$ & $190(46.68)$ & $196(43.55)$ \\
\hline $\begin{array}{l}\text { Breathlessness/ } \\
\text { Shortness of breath }\end{array}$ & $488(56.9)$ & $236(57.98)$ & $252(56)$ \\
\hline Crepitation & $500(58.3)$ & 251 (61.67) & 249 (55.33) \\
\hline Rhonchi & $98(11.4)$ & $53(13.02)$ & $45(10)$ \\
\hline Dyspnoea & $77(9.0)$ & $28(6.88)$ & 49 (10.89) \\
\hline Tachypnea & $74(8.6)$ & 34 (8.35) & $40(8.89)$ \\
\hline Abdominal Pain & $134(15.6)$ & $83(20.39)$ & $51(11.33)$ \\
\hline Facial puffiness & $13(1.5)$ & $10(2.46)$ & $3(0.66)$ \\
\hline Pitting or pedal edema & $92(10.7)$ & $49(12.04)$ & $43(9.55)$ \\
\hline \multicolumn{4}{|l|}{ Co-morbities (n, \%) } \\
\hline $\begin{array}{l}\text { Type } 2 \text { Diabetes } \\
\text { Mellitus }\end{array}$ & $191(22.3)$ & $94(23.1)$ & $97(21.55)$ \\
\hline Hypertension & $216(25.2)$ & $93(22.85)$ & $123(27.33)$ \\
\hline Kidney diseases & $204(23.8)$ & 79 (19.41) & $125(27.78)$ \\
\hline $\begin{array}{l}\text { Cardiovascular } \\
\text { diseases }\end{array}$ & $86(10.0)$ & $30(7.37)$ & $56(12.44)$ \\
\hline Liver diseases & $47(5.5)$ & $7(1.72)$ & $37(8.22)$ \\
\hline $\begin{array}{l}\text { Bronchial asthma/ } \\
\text { COPD }\end{array}$ & $37(4.3)$ & $18(4.42)$ & $19(4.22)$ \\
\hline Respiratory failure & $25(2.9)$ & $10(2.46)$ & $15(3.33)$ \\
\hline Cancer & $23(2.7)$ & $3(0.74)$ & $20(4.44)$ \\
\hline Others & $231(27.0)$ & $113(27.76)$ & $118(26.22)$ \\
\hline \multicolumn{4}{|l|}{ Risk Factors (n, \%) } \\
\hline Pneumonia & $285(33.3)$ & 109 (26.78) & $176(39.11)$ \\
\hline Sepsis & $322(37.6)$ & $73(17.94)$ & $249(55.33)$ \\
\hline Septic Shock & $236(27.5)$ & $50(12.28)$ & $186(41.33)$ \\
\hline AIDS & $7(0.8)$ & $1(0.24)$ & $6(1.33)$ \\
\hline Leptospirosis & $75(8.8)$ & $42(10.32)$ & $33(7.33)$ \\
\hline Scrub typhus & $124(14.5)$ & 107 (26.29) & $17(3.78)$ \\
\hline Dengue & $22(2.6)$ & 19 (4.67) & $3(0.67)$ \\
\hline MODS & $126(14.7)$ & $35(8.60)$ & $91(20.22)$ \\
\hline Poison & $20(2.3)$ & $4(0.98)$ & $16(3.55)$ \\
\hline Burns & $9(1.1)$ & $0(0)$ & $9(2)$ \\
\hline H1N1 & $112(13.1)$ & $50(12.28)$ & $62(13.78)$ \\
\hline Hepatitis & $16(1.9)$ & $8(1.97)$ & $8(1.78)$ \\
\hline Pancreatitis & $61(7.1)$ & $44(10.81)$ & $17(3.78)$ \\
\hline Others & 349 (40.7) & $196(48.16)$ & $153(34)$ \\
\hline
\end{tabular}

SD: Standard deviation; IQR: Interquartile range; COPD: Chronic obstructive pulmonary disease; AIDS: Acquired immunodeficiency syndrome; MODS: Multiple organ dysfunction syndrome.

11977.08), neutrophils count (72.74 \pm 16.93$)$, and erythrocyte sedimentation rate $(43.17 \pm 31.09 \mathrm{~mm} / \mathrm{h})$, were higher than the normal; and the average levels of lymphocytes $(13.23 \pm 10.78 ; n=780)$ were observed to be lesser than the normal (Table 2).

\subsubsection{Arterial blood gas analysis}

An average level of $-5.15 \pm 7.64,19.48 \pm 5.11,18.20 \pm 5.66,27.05$ $\pm 14.80,14.37 \pm 6.97$, and $34.84 \pm 11.70$ for $\mathrm{ABEc}, \mathrm{CHCO} 3$ (st), $\mathrm{CHCO} 3$
Table 2

Laboratory parameters of included participants.

\begin{tabular}{|c|c|c|c|c|}
\hline \multicolumn{5}{|c|}{ Laboratory parameters (Mean, SD) } \\
\hline Parameter & $\begin{array}{l}\text { Normal } \\
\text { range }\end{array}$ & $\begin{array}{l}\text { Total } \\
\text { participants } \\
(\mathrm{N}=857)\end{array}$ & $\begin{array}{l}\text { Recovery } \\
\text { group }(\mathrm{n}= \\
407)\end{array}$ & $\begin{array}{l}\text { Non- } \\
\text { recovery } \\
\text { group }(\mathrm{n}= \\
450)\end{array}$ \\
\hline \multicolumn{5}{|l|}{ Vitals } \\
\hline $\begin{array}{l}\text { Temperature (n } \\
\quad=565)\end{array}$ & $98.6^{\circ} \mathrm{F}$ & $\begin{array}{l}98.82(0.80) \\
{ }^{\circ} \mathrm{F}\end{array}$ & $\begin{array}{l}98.88(0.89) \\
{ }^{\circ} \mathrm{F}\end{array}$ & $\begin{array}{l}98.77(0.67) \\
{ }^{\circ} \mathrm{F}\end{array}$ \\
\hline $\begin{array}{r}\text { Heart rate/Pulse } \\
\text { rate }(\mathrm{n}=724)\end{array}$ & $\begin{array}{l}60-100 \\
\text { bpm }\end{array}$ & $98(22) \mathrm{bpm}$ & 96 (20) bpm & $\begin{array}{l}101(24) \\
\text { bpm }\end{array}$ \\
\hline $\begin{array}{l}\text { Systolic blood } \\
\text { pressure }(\mathrm{n}= \\
724)\end{array}$ & $\begin{array}{l}<120 \\
\mathrm{mmHg}\end{array}$ & $\begin{array}{l}121.49 \\
(25.36) \\
\mathrm{mmHg}\end{array}$ & $\begin{array}{l}121.85 \\
(23.82) \\
\mathrm{mmHg}\end{array}$ & $\begin{array}{l}121.08 \\
(26.96) \\
\mathrm{mmHg}\end{array}$ \\
\hline $\begin{array}{l}\text { Diastolic blood } \\
\text { pressure }(\mathrm{n}= \\
716)\end{array}$ & $\begin{array}{l}<80 \\
\mathrm{mmHg}\end{array}$ & $\begin{array}{l}76.04(12.56) \\
\mathrm{mmHg}\end{array}$ & $\begin{array}{l}76.71 \\
(12.28) \\
\mathrm{mmHg}\end{array}$ & $\begin{array}{l}75.28 \\
(12.83) \\
\mathrm{mmHg}\end{array}$ \\
\hline $\begin{array}{l}\text { Respiratory rate } \\
\quad(\mathrm{n}=500)\end{array}$ & $12-16 \mathrm{bpm}$ & $\begin{array}{l}26.10(8.94) \\
\text { bpm }\end{array}$ & $\begin{array}{l}24.64(7.51) \\
\text { bpm }\end{array}$ & $\begin{array}{l}27.69 \\
(10.03) \mathrm{bpm}\end{array}$ \\
\hline \multicolumn{5}{|l|}{ Blood indices } \\
\hline $\begin{array}{l}\text { Total leukocyte } \\
\quad(\mathrm{n}=846)\end{array}$ & $\begin{array}{l}4000- \\
10000 \\
\text { cells } / \mu \mathrm{L}\end{array}$ & $\begin{array}{l}12408.81 \\
(11977.08) \\
\text { cells } / \mu \mathrm{L}\end{array}$ & $\begin{array}{l}12308.40 \\
(13262.45) \\
\text { cells } / \mu \mathrm{L}\end{array}$ & $\begin{array}{l}12501.02 \\
(10660.15) \\
\text { cells } / \mu \mathrm{L}\end{array}$ \\
\hline $\begin{array}{l}\text { Neutrophils }(\mathrm{n}= \\
\text { 786) }\end{array}$ & $42-74 \%$ & $\begin{array}{l}72.74(16.93) \\
\%\end{array}$ & $\begin{array}{l}72.61 \\
(14.28) \%\end{array}$ & $\begin{array}{l}72.85 \\
(19.09) \%\end{array}$ \\
\hline $\begin{array}{l}\text { Eosinophils }(\mathrm{n}= \\
\text { 478) }\end{array}$ & $1-8 \%$ & $1.04(2.43) \%$ & $1.01(2.62) \%$ & $1.06(2.24) \%$ \\
\hline $\begin{array}{l}\text { Lymphocytes (n } \\
\quad=780 \text { ) }\end{array}$ & $18-44 \%$ & $\begin{array}{l}13.23 \\
(10.78) \%\end{array}$ & $\begin{array}{l}14.57 \\
(10.20) \%\end{array}$ & $\begin{array}{l}11.96 \\
(11.15) \%\end{array}$ \\
\hline $\begin{array}{l}\text { Basophils ( } \mathrm{n}= \\
\text { 418) }\end{array}$ & $1-2 \%$ & $0.57(1.68) \%$ & $0.52(1.40) \%$ & $0.62(1.92) \%$ \\
\hline $\begin{array}{l}\text { Monocytes ( } \mathrm{n}= \\
764 \text { ) }\end{array}$ & $5-13 \%$ & $5.88(4.42) \%$ & $5.95(4.00) \%$ & $5.81(4.79) \%$ \\
\hline $\begin{array}{l}\text { Erythrocyte } \\
\text { Sedimentation } \\
\text { Rate }(n=686)\end{array}$ & $\begin{array}{l}0-20 \mathrm{~mm} / \\
\mathrm{h}\end{array}$ & $\begin{array}{l}43.17 \text { (31.09) } \\
\mathrm{mm} / \mathrm{hr}\end{array}$ & $\begin{array}{l}42.87 \\
(29.99) \mathrm{mm} / \\
\mathrm{hr}\end{array}$ & $\begin{array}{l}43.48 \\
(32.17) \mathrm{mm} / \\
\mathrm{hr}\end{array}$ \\
\hline \multicolumn{5}{|l|}{ Blood gas analysis } \\
\hline $\operatorname{ABEc}(\mathrm{n}=809)$ & - & $-5.15(7.64)$ & $-3.82(6.72)$ & $-6.39(8.21)$ \\
\hline $\begin{array}{l}\mathrm{CHCO} 3 \text { (st) }(\mathrm{n}= \\
823)\end{array}$ & - & $19.48(5.11)$ & $20.47(4.61)$ & $18.55(5.37)$ \\
\hline $\begin{array}{l}\mathrm{CHCO} 3(\mathrm{P})(\mathrm{n}= \\
\quad 825)\end{array}$ & - & $18.20(5.66)$ & $18.95(5.32)$ & $17.50(5.87)$ \\
\hline $\mathrm{CtCO} 2(\mathrm{n}=826)$ & - & $27.05(14.80)$ & $\begin{array}{l}27.97 \\
(14.49)\end{array}$ & $\begin{array}{l}26.18 \\
(15.04)\end{array}$ \\
\hline $\mathrm{CtHb}(\mathrm{n}=825)$ & $\begin{array}{l}13.5-17.5 \\
\mathrm{~g} / \mathrm{dL}\end{array}$ & $\begin{array}{l}11.87(8.40) \\
\mathrm{g} / \mathrm{dL}\end{array}$ & $\begin{array}{l}11.75(7.65) \\
g / d L\end{array}$ & $\begin{array}{l}11.99(9.04) \\
\mathrm{g} / \mathrm{dL}\end{array}$ \\
\hline CtO2e $(\mathrm{n}=827)$ & - & $14.37(6.97)$ & $14.65(5.37)$ & $14.10(8.18)$ \\
\hline Hctc $(\mathrm{n}=803)$ & - & $34.84(11.70)$ & $\begin{array}{l}35.28 \\
(11.76)\end{array}$ & $\begin{array}{l}34.42 \\
(11.63)\end{array}$ \\
\hline $\mathrm{PCO} 2(\mathrm{n}=832)$ & $\begin{array}{l}35-48 \\
\mathrm{mmHg}\end{array}$ & $\begin{array}{l}33.47(15.43) \\
\mathrm{mmHg}\end{array}$ & $\begin{array}{l}32.20 \\
(16.85) \\
\mathrm{mmHg}\end{array}$ & $\begin{array}{l}34.64 \\
(13.87) \\
\mathrm{mmHg}\end{array}$ \\
\hline $\mathrm{PO} 2(\mathrm{n}=831)$ & $\begin{array}{l}\text { 83-109 } \\
\mathrm{mmHg}\end{array}$ & $86.47(52.31)$ & $\begin{array}{l}83.45 \\
(42.47)\end{array}$ & $\begin{array}{l}89.26 \\
(59.85)\end{array}$ \\
\hline $\mathrm{PH}(\mathrm{n}=829)$ & $7.35-7.46$ & $7.59(3.94)$ & $7.60(4.03)$ & $7.59(3.85)$ \\
\hline $\operatorname{SPO} 2(\mathrm{n}=162)$ & $95 \%$ & $\begin{array}{l}84.16(14.93) \\
\%\end{array}$ & $\begin{array}{l}87.12 \\
(10.13) \%\end{array}$ & $\begin{array}{l}81.67 \\
(17.62) \%\end{array}$ \\
\hline $\mathrm{SaO} 2(\mathrm{n}=800)$ & $95-99 \%$ & $\begin{array}{l}89.43(12.99) \\
\%\end{array}$ & $\begin{array}{l}90.97 \\
(10.65) \%\end{array}$ & $\begin{array}{l}87.94 \\
(14.75) \%\end{array}$ \\
\hline
\end{tabular}

bpm: Beats per minute; mmHg: millimetre mercury; mm/hr: millimetre/hour; cells/ $\mu \mathrm{L}:$ cells/microliter; $\mathrm{g} / \mathrm{dL}$ : grams/decilitre.

(P), CtCO2, CtO2e, and Hctc were observed among the included participants, respectively. A lower level of CtHb (11.87 \pm 8.40$)$, PCO2 (33.47 \pm 15.43$)$, SPO2 (84.16 \pm 14.93$)$, and SaO2 (89.43 \pm 12.99$)$ were observed, whereas PO2 (86.47 \pm 52.31$)$ were within the normal and $\mathrm{PH}$ was slightly higher than the normal $(7.59 \pm 3.94)$. The laboratory parameters and arterial blood gas analysis of the included participants are presented in Table 2.

\subsection{Treatment provided to the patients}

Antibiotics were administered in 839 (97.9\%), 401 (98.52\%), and 
$438(97.33 \%)$ of the overall population, recovered patients and nonrecovered patients respectively. Whereas steroids were administered in half of the population, which is $52.3 \%(n=448), 52.82 \%(n=215)$, and $51.78 \%(n=233)$ of the overall population, recovered patients and nonrecovered patients respectively. Among the included participants, 95.3\% $(n=817)$ of the patients were admitted to ICU; 70.1\% $(n=601)$ were taken to the ventilator and $35.1 \%(n=301)$ were given oxygen supplementation (Table 3).

\subsection{Outcomes of ARDS in the patients}

Among the total participants, $47.5 \%(n=407)$ of the population were completely recovered, whereas the remaining $52.5 \%(n=450)$ were not recovered. The average hospitalization days were observed to be 10.08 \pm 7.96 days in the total population, which was more in the recovered group (12.55 \pm 8.16 days) compared to the non-recovered population (7.86 \pm 7.07 days). Similarly, higher average ICU days were observed with the recovered group $(7.22 \pm 6.08$ days $)$ compared to the nonrecovered group ( $6.27 \pm 5.55$ days) where it was $6.72 \pm 5.83$ days in the overall population. Likewise, average ventilation days were higher in the non-recovered group ( $4.50 \pm 5.00$ days) compared to the recovered group ( $3.06 \pm 5.00$ days) where it was $3.82 \pm 5.05$ days in the overall population. The average oxygenation days and antibiotics days were observed to be higher in the recovered group $(1.49 \pm 2.24$ and $12.00 \pm 8.32$ days) compared to the non-recovered group $(0.66 \pm 2.08$ and $7.48 \pm 7.01$ days) where it was $1.05 \pm 2.20$ and $9.63 \pm 7.99$ days in the overall population, respectively. The treatment and outcomes in the included participants are provided in Table 3.

\subsection{Factors affecting the outcomes}

The factors such as scrub typhus (OR:0.138; 95\%CI:0.073-0.263; p $<0.001$ ); dengue (OR:0.183; 95\%CI:0.049-0.679; $\mathrm{p}<0.001$ ); oxygen

Table 3

Treatments and outcomes in the included participants.

\begin{tabular}{|c|c|c|c|}
\hline Parameter & $\begin{array}{l}\text { Total participants } \\
(\mathrm{N}=857)\end{array}$ & $\begin{array}{l}\text { Recovery } \\
\text { group ( }= \\
407)\end{array}$ & $\begin{array}{l}\text { Non-recovery } \\
\text { group }(n=450)\end{array}$ \\
\hline \multicolumn{4}{|c|}{ Treatment Provided (n, \%) } \\
\hline Antibiotics & 839 (97.9) & $401(98.52)$ & $438(97.33)$ \\
\hline Steroids & $448(52.3)$ & $215(52.82)$ & $233(51.78)$ \\
\hline PPI or H2RA & $757(88.3)$ & 362 (88.94) & $395(87.78)$ \\
\hline ICU admission & $817(95.3)$ & $381(93.61)$ & $436(96.89)$ \\
\hline Ventilation & $601(70.1)$ & $226(55.52)$ & $375(83.33)$ \\
\hline Oxygenation & $301(35.1)$ & $209(51.35)$ & $92(20.44)$ \\
\hline \multicolumn{4}{|l|}{ Outcomes } \\
\hline Recovered (n, \%) & $407(47.5)$ & 407 (100) & NA \\
\hline Not recovered (n, \%) & $450(52.5)$ & NA & $450(100)$ \\
\hline $\begin{array}{l}\text { Hospitalization days } \\
\quad \text { (Mean, SD) }\end{array}$ & $10.08(7.96)$ & $12.55(8.16)$ & $7.86(7.07)$ \\
\hline $\begin{array}{l}\text { Hospitalization days } \\
\text { (Median, IQR) }\end{array}$ & $8(5,13)$ & $10(8,15)$ & $6(3,11)$ \\
\hline ICU days (Mean, SD) & $6.72(5.83)$ & $7.22(6.08)$ & $6.27(5.55)$ \\
\hline $\begin{array}{l}\text { ICU days (Median, } \\
\text { IQR) }\end{array}$ & $5(3,9)$ & $6(4,9)$ & $5(2,9)$ \\
\hline $\begin{array}{l}\text { Ventilation days } \\
\text { (Mean, SD) }\end{array}$ & $3.82(5.05)$ & $3.06(5.00)$ & $4.50(5.00)$ \\
\hline $\begin{array}{l}\text { Ventilation days } \\
\text { (Median, IQR) }\end{array}$ & $2(0,5)$ & $1(0,4)$ & $3(1,6)$ \\
\hline $\begin{array}{l}\text { Oxygenation days } \\
\text { (Mean, SD) }\end{array}$ & $1.05(2.20)$ & $1.49(2.24)$ & $0.66(2.08)$ \\
\hline $\begin{array}{l}\text { Oxygenation days } \\
\text { (Median, IQR) }\end{array}$ & $0(0,2)$ & $1(0,2)$ & $0(0,0)$ \\
\hline $\begin{array}{l}\text { Antibiotics days } \\
\text { (Mean, SD) }\end{array}$ & 9.63 (7.99) & $12.00(8.32)$ & $7.48(7.01)$ \\
\hline $\begin{array}{r}\text { Antibiotics days } \\
\text { (Median, IQR) }\end{array}$ & $8(4,12)$ & $10(7,14)$ & $5(3,11)$ \\
\hline
\end{tabular}

PPI: Proton pump inhibitor; H2RA: Histamine 2 receptor antagonist; ICU: intensive care unit; SD: Standard deviation; IQR: Interquartile range. supplementation (OR:0.171; 95\%CI:0.115-0.254; p $<0.001$ ) and pancreatitis (OR:0.458; 95\%CI:0.226-0.927; $\mathrm{p}=0.030$ ) were found to be independent predictors of better recovery. In a univariate analysis, the factors such as age of 60 years or above (OR:2.446; 95\% CI:1.753-3.412; $\mathrm{p}<0.001$ ); male gender (OR:1.543; 95\% CI:1.175-2.028; $\mathrm{p}=0.002$ ); presence of comorbidity (OR:1.586; 95\% CI:1.201-2.095; $\mathrm{p}=0.001$ ); pneumonia (OR:1.756; $95 \%$ CI:1.314-2.346; p < 0.001); sepsis (OR:5.668; 95\%CI:4.140-7.760; p < 0.001); septic shock (OR:5.030; 95\%CI:3.543-7.142; $<<0.001$ ); MODS (OR:2.694; 95\%CI:1.777-4.084; p $<0.001$ ); poison exposure (OR:3.714; 95\%CI:1.231-11.203; $\mathrm{p}=0.020$ ); cardiovascular diseases (OR:1.786; 95\%CI:1.121-2.845; $\mathrm{p}=0.015$ ); liver diseases (OR:5.575; 95\%CI:2.468-12.591; $\mathrm{p}<0.001$ ); cancer (OR:3.714; 95\% CI:1.231-11.203; $\mathrm{p}=0.020$ ); ICU admission (OR:2.125; 95\% CI:1.094-4.129; $\mathrm{p}=0.026$ ); and ventilation requirement (OR:4.004; 95\%CI:2.920-5.491; $\mathrm{p}<0.001$ ) were significantly associated with a higher risk of non-recovery, Whereas, in a multi variate analysis, the factors such as age of 60 years or above (OR:2.120; 95\%CI:1.359-3.306; $\mathrm{p}<0.001$ ); sepsis (OR:2.600; 95\%CI:1.701-3.972; $\mathrm{p}<0.001$ ); septic shock (OR:2.351; 95\%CI:1.478-3.739; $\mathrm{p}<0.001$ ); liver diseases (OR:3.319; 95\%CI:1.284-8.578; $\mathrm{p}=0.013$ ) and ventilation requirement (OR:3.792; 95\%CI:2.481-5.795; p < 0.001) were observed to be the independent predictors of non-recovery in ARDS patients. The univariate and multi variate analysis on factors affecting non-recovery and recovery is provided in Table 4.

\section{Discussion}

ARDS is still a global concern with its high mortality and challenges in management even in this modern era of medicine. Currently, the severe acute respiratory syndrome coronavirus 2 (SARS-CoV 2 or COVID-19) became another threat to the whole world which changed the complete face of the world. ${ }^{10}$ In our study, $57.9 \%$ were male and $42.1 \%$ were females, respectively. This was similar to the findings of the Indian studies conducted by Sharma SK et al., and Prabhu et al., in which they reported $60.9 \%$ and $60.7 \%$ male proportion, respectively. ${ }^{5,11}$ Our study population had an average age of 46.86 years, which was apparent to the findings of other south Indian studies. ${ }^{4,11}$ Also, we observed a recovery rate of $47.49 \%$, which was almost near to the findings of other Indian studies. ${ }^{4,5,11}$

The mean age for non-recovered patients was higher $(49.08 \pm 16.57$ years) than the recovered patients ( $44.41 \pm 14.53$ years). Moreover, age greater than 60 years appeared to be an independent predictor of nonrecovery in our group of patients. Similarly, numerous studies across the world observed that advanced age is an independent predictor of non-recovery or mortality in ARDS patients. ${ }^{7,12-14}$ Moreover, some studies interpreted that, younger age is an independent predictor of better recovery. ${ }^{15,16}$

There were 496 (57.9\%) males and 361 (42.1\%) females in our study, with similar male domination in recovery $(52.34 \%$ vs $47.66 \%)$ and non-recovery $(62.89 \%$ vs $37.11 \%)$. Similar male domination was observed in studies from Pakistan, ${ }^{17}$ India, ${ }^{5}$ and other parts of the world. ${ }^{12,15,18,19}$ Being a male gender significantly affected the non-recovery in univariate analysis $(P=0.002)$, whereas, it was not an independent predictor $(\mathrm{P}=0.066)$ in our study. Similar conflicting findings were observed in the previous studies, in which some studies identified that being a male gender is a risk factor for non-recovery or mortality among ARDS patients, ${ }^{12-14}$ whereas some studies didn't. ${ }^{16,18,19}$

Sepsis, pneumonia, septic shock, and MODS were the major aetiological reasons for ARDS in our population. Pneumonia, sepsis, septic shock, MODS, poisoning were significant aetiological conditions that contributed to the non-recovery in our patients, in which sepsis and septic shock were the independent predictors of non-recovery. Similar findings were reported in other studies where they recorded that, sepsis, MODS, and refractory shock were the leading causes of death., ${ }^{5,17}$ 
Table 4

Univariate and multi-variate analysis on factors affecting the outcomes.

\begin{tabular}{|c|c|c|c|c|}
\hline \multirow[t]{2}{*}{ VARIABLE } & \multicolumn{2}{|c|}{ UNIVARIATE ANALYSIS } & \multicolumn{2}{|c|}{ MULTIVARIATE ANALYSIS } \\
\hline & $\begin{array}{l}\text { Unadjusted odds } \\
\text { ratio }(95 \% \mathrm{CI})\end{array}$ & P-value & $\begin{array}{l}\text { Adjusted odds } \\
\text { ratio }(95 \% \mathrm{CI})\end{array}$ & P-value \\
\hline \multicolumn{5}{|l|}{ Age group } \\
\hline $\begin{array}{l}<60 \text { years } \\
(n=652)\end{array}$ & 1 & NA & $\begin{array}{l}2.120 \\
(1.359-3.306)\end{array}$ & $0.001^{\mathrm{a}}$ \\
\hline $\begin{array}{l}60 \text { or above } \\
(n=205)\end{array}$ & $\begin{array}{l}2.446 \\
(1.753-3.412)\end{array}$ & $<0.001^{\mathrm{a}}$ & & \\
\hline \multicolumn{5}{|l|}{ Gender } \\
\hline $\begin{array}{l}\text { Female (n } \\
=361)\end{array}$ & 1 & NA & $\begin{array}{l}1.398 \\
(0.968-2.019)\end{array}$ & 0.074 \\
\hline $\begin{array}{l}\text { Male }(n= \\
496)\end{array}$ & $\begin{array}{l}1.543 \\
(1.175-2.028)\end{array}$ & $0.002^{\mathrm{a}}$ & & \\
\hline \multicolumn{5}{|c|}{ Smoking status } \\
\hline $\begin{array}{l}\text { Not } \\
\text { reported (n } \\
=431)\end{array}$ & 1 & NA & NA & \\
\hline $\begin{array}{l}\text { No }(n= \\
393)\end{array}$ & $\begin{array}{l}0.880 \\
(0.669-1.157)\end{array}$ & 0.360 & & \\
\hline $\begin{array}{l}\text { Yes }(\mathrm{n}= \\
33)\end{array}$ & $\begin{array}{l}1.164 \\
(0.569-2.382)\end{array}$ & 0.677 & & \\
\hline \multicolumn{5}{|c|}{ Alcoholic status } \\
\hline $\begin{array}{l}\text { Not } \\
\text { reported (n } \\
=422)\end{array}$ & 1 & NA & NA & \\
\hline $\begin{array}{l}\text { No }(n= \\
352)\end{array}$ & $\begin{array}{l}0.852 \\
(0.642-1.132)\end{array}$ & 0.270 & & \\
\hline $\begin{array}{l}\text { Yes }(\mathrm{n}= \\
83)\end{array}$ & $\begin{array}{l}0.951 \\
(0.593-1.524)\end{array}$ & 0.834 & & \\
\hline \multicolumn{5}{|c|}{ Presence of co-morbidity } \\
\hline $\begin{array}{l}\text { No }(\mathrm{n}= \\
322)\end{array}$ & 1 & NA & $\begin{array}{l}0.708 \\
(0.477-1.051)\end{array}$ & 0.087 \\
\hline $\begin{array}{l}\text { Yes }(\mathrm{n}= \\
535)\end{array}$ & $\begin{array}{l}1.586 \\
(1.201-2.095)\end{array}$ & $0.001^{\mathrm{a}}$ & & \\
\hline \multicolumn{5}{|l|}{ Risk factors } \\
\hline \multicolumn{5}{|l|}{ Pneumonia } \\
\hline $\begin{array}{l}\text { No }(\mathrm{n}= \\
572)\end{array}$ & 1 & NA & $\begin{array}{l}1.119 \\
(0.761-1.647)\end{array}$ & 0.568 \\
\hline $\begin{array}{l}\text { Yes }(\mathrm{n}= \\
285)\end{array}$ & $\begin{array}{l}1.756 \\
(1.314-2.346)\end{array}$ & $<0.001^{\mathrm{a}}$ & & \\
\hline \multicolumn{5}{|l|}{ Sepsis } \\
\hline $\begin{array}{l}\text { No }(\mathrm{n}= \\
535)\end{array}$ & 1 & NA & $\begin{array}{l}2.600 \\
(1.701-3.972)\end{array}$ & $<0.001^{\mathrm{a}}$ \\
\hline $\begin{array}{l}\text { Yes }(\mathrm{n}= \\
322)\end{array}$ & $\begin{array}{l}5.668 \\
(4.140-7.760)\end{array}$ & $<0.001^{\mathrm{a}}$ & & \\
\hline \multicolumn{5}{|l|}{ Septic Shock } \\
\hline $\begin{array}{l}\text { No }(\mathrm{n}= \\
621)\end{array}$ & 1 & NA & $\begin{array}{l}2.351 \\
(1.478-3.739)\end{array}$ & $<0.001^{\mathrm{a}}$ \\
\hline $\begin{array}{l}\text { Yes }(\mathrm{n}= \\
236)\end{array}$ & $\begin{array}{l}5.030 \\
(3.543-7.142)\end{array}$ & $<0.001^{\mathrm{a}}$ & & \\
\hline \multicolumn{5}{|c|}{ Acquired immunodeficiency syndrome } \\
\hline $\begin{array}{l}\text { No }(\mathrm{n}= \\
851)\end{array}$ & 1 & NA & NA & \\
\hline Yes $(n=6)$ & $\begin{array}{l}4.562 \\
(0.531-39.211)\end{array}$ & 0.167 & & \\
\hline \multicolumn{5}{|l|}{ Leptospirosis } \\
\hline $\begin{array}{l}\text { No }(\mathrm{n}= \\
782)\end{array}$ & 1 & NA & NA & \\
\hline $\begin{array}{l}\text { Yes }(\mathrm{n}= \\
75)\end{array}$ & $\begin{array}{l}0.688 \\
(0.427-1.108)\end{array}$ & 0.124 & & \\
\hline \multicolumn{5}{|l|}{ Scrub typhus } \\
\hline $\begin{array}{l}\text { No }(n= \\
733)\end{array}$ & 1 & NA & $\begin{array}{l}0.138 \\
(0.073-0.263)\end{array}$ & $<0.001^{\mathrm{a}}$ \\
\hline $\begin{array}{l}\text { Yes }(\mathrm{n}= \\
124)\end{array}$ & $\begin{array}{l}0.110 \\
(0.065-0.187)\end{array}$ & $<0.001^{\mathrm{a}}$ & & \\
\hline \multicolumn{5}{|l|}{ Dengue } \\
\hline $\begin{array}{l}\text { No }(\mathrm{n}= \\
835)\end{array}$ & 1 & NA & $\begin{array}{l}0.183 \\
(0.049-0.679)\end{array}$ & $<0.001^{\mathrm{a}}$ \\
\hline $\begin{array}{l}\text { Yes }(\mathrm{n}= \\
22)\end{array}$ & $\begin{array}{l}0.137 \\
(0.040-0.467)\end{array}$ & $0.001^{\mathrm{a}}$ & & \\
\hline \multicolumn{5}{|c|}{ Multiple organ dysfunction syndrome } \\
\hline $\begin{array}{l}\text { No }(\mathrm{n}= \\
731)\end{array}$ & 1 & NA & $\begin{array}{l}1.260 \\
(0.746-2.127)\end{array}$ & 0.388 \\
\hline $\begin{array}{l}\text { Yes }(n= \\
126)\end{array}$ & $\begin{array}{l}2.694 \\
(1.777-4.084)\end{array}$ & $<0.001^{\mathrm{a}}$ & & \\
\hline
\end{tabular}

Table 4 (continued)

\begin{tabular}{|c|c|c|c|c|}
\hline \multirow[t]{2}{*}{ VARIABLE } & \multicolumn{2}{|c|}{ UNIVARIATE ANALYSIS } & \multicolumn{2}{|c|}{ MULTIVARIATE ANALYSIS } \\
\hline & $\begin{array}{l}\text { Unadjusted odds } \\
\text { ratio }(95 \% \mathrm{CI})\end{array}$ & P-value & $\begin{array}{l}\text { Adjusted odds } \\
\text { ratio }(95 \% \mathrm{CI})\end{array}$ & P-value \\
\hline \multicolumn{5}{|l|}{ Poisoning } \\
\hline $\begin{array}{l}\text { No ( } \mathrm{n}= \\
837)\end{array}$ & 1 & NA & $\begin{array}{l}3.203 \\
(0.902-11.373)\end{array}$ & 0.072 \\
\hline $\begin{array}{l}\text { Yes }(\mathrm{n}= \\
20)\end{array}$ & $\begin{array}{l}3.714 \\
(1.231-11.203)\end{array}$ & $0.020^{\mathrm{a}}$ & & \\
\hline \multicolumn{5}{|l|}{ H1N1 } \\
\hline $\begin{array}{l}\text { No }(\mathrm{n}= \\
745)\end{array}$ & 1 & NA & NA & \\
\hline $\begin{array}{l}\text { Yes }(\mathrm{n}= \\
112)\end{array}$ & $\begin{array}{l}1.141 \\
(0.765-1.701)\end{array}$ & 0.518 & & \\
\hline \multicolumn{5}{|l|}{ Hepatitis } \\
\hline $\begin{array}{l}\text { No ( } \mathrm{n}= \\
841)\end{array}$ & 1 & NA & NA & \\
\hline $\begin{array}{l}\text { Yes }(n= \\
16)\end{array}$ & $\begin{array}{l}0.903 \\
(0.336-2.428)\end{array}$ & 0.839 & & \\
\hline \multicolumn{5}{|l|}{ Pancreatitis } \\
\hline $\begin{array}{l}\text { No }(\mathrm{n}= \\
796)\end{array}$ & 1 & NA & $\begin{array}{l}0.458 \\
(0.226-0.927)\end{array}$ & $0.030^{\mathrm{a}}$ \\
\hline $\begin{array}{l}\text { Yes }(\mathrm{n}= \\
61)\end{array}$ & $\begin{array}{l}0.324 \\
(0.182-0.577)\end{array}$ & $<0.001^{\mathrm{a}}$ & & \\
\hline \multicolumn{5}{|c|}{ Co-morbidities } \\
\hline \multicolumn{5}{|c|}{ Type-2-dibates mellitus } \\
\hline $\begin{array}{l}\text { No }(n= \\
666)\end{array}$ & 1 & NA & NA & \\
\hline $\begin{array}{l}\text { Yes }(n= \\
191)\end{array}$ & $\begin{array}{l}0.915 \\
(0.663-1.262)\end{array}$ & 0.589 & & \\
\hline \multicolumn{5}{|l|}{ Hypertension } \\
\hline $\begin{array}{l}\text { No }(n= \\
641)\end{array}$ & 1 & NA & NA & \\
\hline $\begin{array}{l}\text { Yes }(n= \\
216)\end{array}$ & $\begin{array}{l}1.270 \\
(0.931-1.733)\end{array}$ & 0.132 & & \\
\hline \multicolumn{5}{|c|}{ Kidney diseases } \\
\hline $\begin{array}{l}\text { No }(n= \\
666)\end{array}$ & 1 & NA & NA & \\
\hline $\begin{array}{l}\text { AKI }(n= \\
168)\end{array}$ & $\begin{array}{l}0.635 \\
(0.271-1.488)\end{array}$ & 0.296 & & \\
\hline $\begin{array}{l}\text { Yes }(n= \\
23)\end{array}$ & $\begin{array}{l}1.071 \\
(0.438-2.619)\end{array}$ & 0.880 & & \\
\hline \multicolumn{5}{|c|}{ Cardiovascular disease } \\
\hline $\begin{array}{l}\text { No }(\mathrm{n}= \\
771)\end{array}$ & 1 & NA & $\begin{array}{l}1.798 \\
(0.962-3.358)\end{array}$ & 0.066 \\
\hline $\begin{array}{l}\text { Yes }(n= \\
86)\end{array}$ & $\begin{array}{l}1.786 \\
(1.121-2.845)\end{array}$ & $0.015^{\mathrm{a}}$ & & \\
\hline \multicolumn{5}{|l|}{ Liver Diseases } \\
\hline $\begin{array}{l}\text { No }(\mathrm{n}= \\
810)\end{array}$ & 1 & NA & $\begin{array}{l}3.319 \\
(1.284-8.578)\end{array}$ & $0.013^{\mathrm{a}}$ \\
\hline $\begin{array}{l}\text { Yes }(n= \\
47)\end{array}$ & $\begin{array}{l}5.575 \\
(2.468-12.591)\end{array}$ & $<0.001^{\mathrm{a}}$ & & \\
\hline \multicolumn{5}{|c|}{ Bronchial asthma/Chronic obstructive pulmonary disease } \\
\hline $\begin{array}{l}\text { No }(\mathrm{n}= \\
820)\end{array}$ & 1 & NA & NA & \\
\hline $\begin{array}{l}\text { Yes }(\mathrm{n}= \\
37)\end{array}$ & $\begin{array}{l}0.953 \\
(0.493-1.842)\end{array}$ & 0.885 & & \\
\hline \multicolumn{5}{|c|}{ Respiratory failure } \\
\hline $\begin{array}{l}\text { No }(\mathrm{n}= \\
832)\end{array}$ & 1 & NA & NA & \\
\hline $\begin{array}{l}\text { Yes }(n= \\
25)\end{array}$ & $\begin{array}{l}1.369 \\
(0.608-3.082)\end{array}$ & 0.448 & & \\
\hline \multicolumn{5}{|l|}{ Cancer } \\
\hline $\begin{array}{l}\text { No }(\mathrm{n}= \\
837)\end{array}$ & 1 & $0.020^{\mathrm{a}}$ & $\begin{array}{l}3.134 \\
(0.829-11.847)\end{array}$ & 0.092 \\
\hline $\begin{array}{l}\text { Yes }(\mathrm{n}= \\
20)\end{array}$ & $\begin{array}{l}3.714 \\
(1.231-11.203)\end{array}$ & & & \\
\hline \multicolumn{5}{|c|}{ Treatment provided } \\
\hline \multicolumn{5}{|c|}{ Antibiotics use } \\
\hline No $(\mathrm{n}=18)$ & 1 & 0.231 & NA & \\
\hline $\begin{array}{l}\text { Yes }(\mathrm{n}= \\
839)\end{array}$ & $\begin{array}{l}0.546 \\
(0.203-1.469)\end{array}$ & & & \\
\hline \multicolumn{5}{|l|}{ Steroid use } \\
\hline $\begin{array}{l}\text { No (n = } \\
409)\end{array}$ & 1 & NA & NA & \\
\hline $\begin{array}{l}\text { Yes }(n= \\
448)\end{array}$ & $\begin{array}{l}0.959 \\
(0.733-1.254)\end{array}$ & 0.759 & & \\
\hline
\end{tabular}


Table 4 (continued)

\begin{tabular}{|c|c|c|c|c|}
\hline \multirow[t]{2}{*}{ VARIABLE } & \multicolumn{2}{|c|}{ UNIVARIATE ANALYSIS } & \multicolumn{2}{|c|}{ MULTIVARIATE ANALYSIS } \\
\hline & $\begin{array}{l}\text { Unadjusted odds } \\
\text { ratio }(95 \% \mathrm{CI})\end{array}$ & P-value & $\begin{array}{l}\text { Adjusted odds } \\
\text { ratio }(95 \% \mathrm{CI})\end{array}$ & P-value \\
\hline \multicolumn{5}{|c|}{ Intensive Care Unit admission } \\
\hline No $(n=40)$ & 1 & $0.026^{\mathrm{a}}$ & 1.816 & 0.157 \\
\hline $\begin{array}{l}\text { Yes }(\mathrm{n}= \\
817)\end{array}$ & $\begin{array}{l}2.125 \\
(1.094-4.129)\end{array}$ & & $(0.795-4.151)$ & \\
\hline \multicolumn{5}{|c|}{ Ventilation requirement } \\
\hline $\begin{array}{l}\text { No }(\mathrm{n}= \\
256)\end{array}$ & 1 & $<0.001^{\mathrm{a}}$ & $\begin{array}{l}3.792 \\
(2.481-5.795)\end{array}$ & $<0.001^{\mathrm{a}}$ \\
\hline $\begin{array}{l}\text { Yes }(\mathrm{n}= \\
601)\end{array}$ & $\begin{array}{l}4.004 \\
(2.920-5.491)\end{array}$ & & & \\
\hline \multicolumn{5}{|l|}{ Oxygenation } \\
\hline $\begin{array}{l}\text { No }(\mathrm{n}= \\
556)\end{array}$ & 1 & $<0.001^{\mathrm{a}}$ & $\begin{array}{l}0.171 \\
(0.115-0.254)\end{array}$ & $<0.001^{\mathrm{a}}$ \\
\hline $\begin{array}{l}\text { Yes }(n= \\
301)\end{array}$ & $\begin{array}{l}0.243 \\
(0.180-0.329)\end{array}$ & & & \\
\hline
\end{tabular}

NA: Not applicable.

a Indicates the significant effect.

Pneumonia was found to be a predominant risk factor for ARDS and sepsis is the connecting link between pneumonia and ARDS. ${ }^{20}$

The comorbidities such as kidney diseases, cardiovascular diseases, liver diseases, and cancer significantly affected the non-recovery, among liver diseases was an independent predictor. A similar observation regarded to the liver disease and worsening of ARDS was recorded by many studies. ${ }^{7,21}$ Interestingly, many studies observed that ARDS is usual comorbidity in liver disease patients, which further leads to a worse prognosis or death. ${ }^{22-24}$ This might be due to many associated conditions such as hepato-pulmonary syndrome, Porto-pulmonary hypertension, hepatic hydrothorax, and related hypoxemia. ${ }^{23}$ Similarly, the patients who underwent a recent cardiovascular surgery, were more prone to develop ARDS and its further complications ${ }^{25}$ though there is conflicting result on the impact of cardiovascular problems on mortality in ARDS patients. ${ }^{25-27}$

The ICU admission and ventilation requirement was significantly associated with non-recovery in which ventilation requirement was observed to be an independent predictor in our population. Evidence indicates that wide use of ventilation is associated with higher mortality in ARDS. ${ }^{28,29}$ However, the factors such as scrub typhus, dengue, and oxygen supplementation were independent predictors of a better recovery. This might be due to the lesser severity and complication of the scrub typhus and dengue, which ultimately lead to a better recovery. Moreover, oxygen supplementation will be helpful to improve oxygen saturation which helps for better survival.

The antibiotics and steroids were used in $97.9 \%$ and $52.3 \%$ of the population, respectively, which was similar to the findings of the study conducted by Prabhu VA et al., where they reported $98.6 \%$ of antibiotic use and $48.6 \%$ of steroids use. ${ }^{11}$ Though the use of antibiotics and steroids were observed to be clinically effective and associated with a better recovery rate, it was not significant. The use of steroids is highly controversial in ARDS concerning the onset of administration, dose, route of administration, and duration of treatment. Khan $\mathrm{S}$ et al. recorded the controversial effect of systemic corticosteroids in the management of ARDS, COVID-19, and Middle East Respiratory Syndrome due to their increased mortality and a higher likelihood of invasive mechanical ventilation. $^{10}$

Drug utilization evaluations are the best way of method to estimate the use and effect of different types of medications used for a clinical condition. ${ }^{30,31}$ Hence, through assessing the use of antibiotics and steroids for the ARDS, it will be helpful to come up with further antibiotics policies and treatment guidelines. A previous study from our setting by Magazine $\mathrm{R}$ et al. recorded that all ARDS patients were prescribed antibiotics and $41.3 \%$ were prescribed corticosteroids. ${ }^{32}$

Additionally, a very recent overview of meta-analyses by Rashid M et al. reported inconsistent evidence on the mortality reduction in the steroid group among the ARDS patients. Their findings indicate significant mortality reduction in the observations of recent meta-analyses of randomized controlled trials (RCTs), whereas older studies are observed to have a non-significant effect. Moreover, an updated meta-analysis by the same researchers found a significant mortality reduction in the pooled estimation of RCTs, but not in cohort studies. ${ }^{33}$

Though our study has the disadvantage of being retrospective, a significant portion of the sample will be useful in deriving appropriate conclusion. Lack of information due to retrospective study design, we had to exclude samples (negligible amount $<20$ ). The results of this study will help in the development of a tool or model for predicting mortality and treatment outcomes in ARDS patients. Further, wellcontrolled prospective studies are needed to assess the effect of steroids in ARDS patients. Additionally, location-based-treatment guidelines needs to be devised to reduce mortality from ARDS.

\section{Conclusion}

The non-recovery rate from ARDS is still observed to be more than the cure rate even in presence of this modern system of medicine. The patients with scrub typhus, dengue, and oxygen administration were observed to have better survival. Whereas, the factors such as advanced age, sepsis, septic shock, liver diseases, and ventilation requirements were the independent predictors of non-recovery in our population. Though the use of antibiotics and steroids improved the recovery, it was not statistically significant.

\section{Funding}

No funding is received for this study.

\section{Authorship statement}

Conception and design of study: Muhammed Rashid (MR), Girish Thunga (GT), Sreedharan Nair (SN); Acquisition of data: MR; Analysis and/or interpretation of data: MR, Vijayanarayana Kunhikatta (VK), Viji Pulikkel Chandran (VPC); Drafting the manuscript: MR; Clinical Inputs to the draft: Vishal Shanbhag (VS), Raviraja V Acharya (RVA), GT; Revising the manuscript: Manasvini Ramakrishnan (RM), Deepa Sudalai Muthu (DSM), VK, SN, VS; Approval of the version of the manuscript to be published: MR, RM, DSM, VPC, GT, VK, VS, RVA, SN.

\section{Data availability statement}

The related to this work is not deposited in any repository. It will be available from the corresponding author on appropriate request.

\section{Ethical committee statement}

This retrospective study was carried out after obtaining ethical approval from the relevant institutional ethics committee (IEC 549/ 2019).

\section{Declaration of competing interest}

All authors report no conflicts of interest to declare. The listed authors are solely responsible for the originality and content of the manuscript. The authors have no relevant affiliations or financial involvement with any organization or entity with a financial interest in or financial conflict with the subject matter or materials discussed in the manuscript.

\section{Acknowledgement}

Muhammed Rashid would like to acknowledge DST-INSPIRE Fellowship, Department of Science and Technology, Government of 
India, New Delhi, India [DST/INSPIRE Fellowship/2019/IF190205] for awarding the fellowship for his doctoral studies (Ph.D). The authors would like to thank the Manipal Academy of Higher Education (MAHE), Manipal College of Pharmaceutical Sciences (MCOPS), Kasturba Medical College (KMC), Manipal and Department of Pharmacy Practice for the library facilities and the best possible completion of this work.

\section{References}

1 Bernard GR, Artigas A, Brigham KL, et al. The American-European Consensus Conference on ARDS. Definitions, mechanisms, relevant outcomes, and clinical trial coordination. Am J Respir Crit Care Med. 1994;149(3 Pt 1):818-824. https://doi.org/ 10.1164/ajrccm.149.3.7509706.

2 Ranieri VM, Rubenfeld GD, Thompson BT, et al. Acute respiratory distress syndrome: the Berlin Definition. JAMA. 2012;307(23):2526-2533. https://doi.org/10.1001/ jama.2012.5669.

3 Confalonieri M, Salton F, Fabiano F. Acute respiratory distress syndrome. Eur Respir Rev. 2017;26(144). https://doi.org/10.1183/16000617.0116-2016.

4 Singh G, Gladdy G, Chandy TT, Sen N. Incidence and outcome of acute lung injury and acute respiratory distress syndrome in the surgical intensive care unit. Indian $J$ Crit Care Med. 2014;18(10):659-665. https://doi.org/10.4103/0972-5229.142175.

5 Sharma SK, Gupta A, Biswas A, et al. Aetiology, outcomes \& predictors of mortality in acute respiratory distress syndrome from a tertiary care centre in north India. Indian J Med Res. 2016;143(6):782-792. https://doi.org/10.4103/09715916.192063.

6 Matthay MA, Zemans RL, Zimmerman GA, et al. Acute respiratory distress syndrome. Nat Rev Dis Prim. 2019;5(1):18. https://doi.org/10.1038/s41572-019-0069-0.

7 Seeley E, McAuley DF, Eisner M, Miletin M, Matthay MA, Kallet RH. Predictors of mortality in acute lung injury during the era of lung protective ventilation. Thorax. 2008;63(11):994-998. https://doi.org/10.1136/thx.2007.093658.

8 Kao KC, Hsieh MJ, Lin SW, et al. Survival predictors in elderly patients with acute respiratory distress syndrome: a prospective observational cohort study. Sci Rep. 2018;8(1):13459. https://doi.org/10.1038/s41598-018-31811-w.

9 IBM Corp. Released. IBM SPSS Statistics for Windows, Version 20.0. Armonk, NY: IBM Corp; 2011.

10 Khan S, Gionfriddo MR, Cortes-Penfield N, Thunga G, Rashid M. The trade-off dilemma in pharmacotherapy of COVID-19: systematic review, meta-analysis, and implications. Expet Opin Pharmacother. 2020;21(15):1821-1849. https://doi.org/ 10.1080/14656566.2020.1792884.

11 Prabhu VA, Shivanshu A, Rithu S, et al. Efficacy of corticosteroids in acute respiratory distress Syndrome: an observational study. J Young Pharm. 2017;9(1):65. https://doi.org/10.5530/jyp.2017.9.13.

12 Gong MN, Thompson BT, Williams P, Pothier L, Boyce PD, Christiani DC. Clinical predictors of and mortality in acute respiratory distress syndrome: potential role of red cell transfusion. Crit Care Med. 2005;33(6):1191-1198. https://doi.org/10.1097/ 01.ccm.0000165566.82925.14.

13 Luhr OR, Antonsen K, Karlsson M, et al. Incidence and mortality after acute respiratory failure and acute respiratory distress syndrome in Sweden, Denmark, and Iceland. The ARF Study Group. Am J Respir Crit Care Med. 1999;159(6):1849-1861. https://doi.org/10.1164/ajrccm.159.6.9808136.

14 Rubenfeld GD, Caldwell E, Peabody E, et al. Incidence and outcomes of acute lung injury. NEJM. 2005;353(16):1685-1693. https://doi.org/10.1056/NEJMc053159.

15 Luecke T, Muench E, Roth $\mathrm{H}$, et al. Predictors of mortality in ARDS patients referred to a tertiary care centre: a pilot study. Eur J Anaesthesiol. 2006;23(5):403-410. https://doi.org/10.1017/s0265021505001870.

16 Kao K-C, Hsieh M-J, Lin S-W, et al. Survival predictors in elderly patients with acute respiratory distress syndrome: a prospective observational cohort study. Sci Rep. 2018;8(1):13459. https://doi.org/10.1038/s41598-018-31811-w.
17 Sharif N, Irfan M, Hussain J, Khan J. Factors associated within 28 days in-hospital mortality of patients with acute respiratory distress syndrome. BioMed Res Int. 2013; 2013:564547. https://doi.org/10.1155/2013/564547.

18 McNicholas BA, Madotto F, Pham T, et al. Demographics, management and outcome of females and males with acute respiratory distress syndrome in the LUNG SAFE prospective cohort study. Eur Respir J. 2019;54(4). https://doi.org/10.1183/ 13993003.00609-2019.

19 Li S, Zhao D, Cui J, Wang L, Ma X, Li Y. Prevalence, potential risk factors and mortality rates of acute respiratory distress syndrome in Chinese patients with sepsis. J Int Med Res. 2020;48(2). https://doi.org/10.1177/0300060519895659, 300060519895659 .

20 Bauer TT, Ewig S, Rodloff AC, Müller EE. Acute respiratory distress syndrome and pneumonia: a comprehensive review of clinical data. Clin Infect Dis. 2006;43(6): 748-756. https://doi.org/10.1086/506430.

21 Dizier S, Forel JM, Ayzac L, et al. Early hepatic dysfunction is associated with a worse outcome in patients presenting with acute respiratory distress syndrome: a post-hoc analysis of the ACURASYS and PROSEVA studies. PLOS One. 2015;10(12), e0144278. https://doi.org/10.1371/journal.pone.0144278.

22 Boente RD, Sheikh A, Bosslet GT, Ghabril MS. Outcomes of acute respiratory distress syndrome in mechanically ventilated patients with cirrhosis. Crit Care Explor. 2019;1 (9), e0040. https://doi.org/10.1097/cce.0000000000000040.

23 Karcz M, Bankey B, Schwaiberger D, Lachmann B, Papadakos PJ. Acute respiratory failure complicating advanced liver disease. Semin Respir Crit Care Med. 2012;33(1): 96-110. https://doi.org/10.1055/s-0032-1301738.

24 Yang P, Formanek P, Scaglione S, Afshar M. Risk factors and outcomes of acute respiratory distress syndrome in critically ill patients with cirrhosis. Hepatol Res. 2019;49(3):335-343. https://doi.org/10.1111/hepr.13240.

25 Hoegl S, Zwissler B, Eltzschig HK, Vohwinkel C. Acute respiratory distress syndrome following cardiovascular surgery: current concepts and novel therapeutic approaches. Curr Opin Anaesthesiol. 2016;29(1):94-100. https://doi.org/10.1097/ aco.0000000000000283.

26 Vieillard-Baron A, Girou E, Valente E, et al. Predictors of mortality in acute respiratory distress syndrome. Focus on the role of right heart catheterization. Am J Respir Crit Care Med. 2000;161(5):1597-1601.

27 Fuller BM, Mohr NM, Graetz TJ, et al. The impact of cardiac dysfunction on acute respiratory distress syndrome and mortality in mechanically ventilated patients with severe sepsis and septic shock: an observational study. J Crit Care. 2015;30(1):65-70. https://doi.org/10.1016/j.jcrc.2014.07.027.

28 Máca J, Jor O, Holub M, et al. Past and present ARDS mortality rates: a systematic review. Respir Care. 2017;62(1):113-122. https://doi.org/10.4187/respcare.04716.

29 Brower RG, Matthay MA, Morris A, Schoenfeld D, Thompson BT, Wheeler A. Ventilation with lower tidal volumes as compared with traditional tidal volumes for acute lung injury and the acute respiratory distress syndrome. NEJM. 2000;342(18): 1301-1308. https://doi.org/10.1056/nejm200005043421801.

30 Venkataraman R, Rashid M, Shashikantha B, et al. Prescribing pattern of antihypertensive medication and adherence to Joint National Commission-8 guidelines in a rural tertiary care Indian teaching hospital. $J$ Basic Clin Physiol Pharmacol. 2019;31(1). https://doi.org/10.1515/jbcpp-2019-0133.

31 Chhabra M, Jangra MS, Rashid M, Sharma P, Vidyasagar K. Pattern, severity, and outcome of adverse drug reactions in a tertiary care hospital: an evidence from a cross-sectional study. J Basic Clin Physiol Pharmacol. 2021. https://doi.org/10.1515/ jbcpp-2020-0218.

32 Magazine R, Rao S, Chogtu B. Prescribing patterns of drugs in acute respiratory distress syndrome (ARDS): an observational study. J Clin Diagn Res. 2015;9(2): FC01-4. https://doi.org/10.7860/jcdr/2015/10411.5519.

33 Rashid M, Khan S, Datta D, et al. Efficacy and safety of corticosteroids in acute respiratory distress syndrome: an overview of meta-analyses. Int J Clin Pract. 2021, e14645. https://doi.org/10.1111/ijcp.14645. 